\section{Nano/microwires of coronene for sensing electron-deficient aromatics}

\author{
Hong Wang, Xiao-He Xu, Hai-Feng Ji \\ Department of Chemistry, Drexel \\ University, Philadelphia, USA
}

\begin{abstract}
We report the synthesis, characterization, and application of coronene nano/microwires for sensing electron-deficient aromatics. The coronene nano/microwires were prepared by a vapor deposition method. The nano /microwires were approximately $300 \mathrm{~nm}$ in diameter and 3-5 $\mu \mathrm{m}$ long. ATR-IR spectroscopy and TG/DTA showed that coronene remains intact and does not undergo polymerization or decomposition during the vapor deposition. Cross-optical polarized microscopy showed a birefringent crystal structure of the coronene microwires. Fluorescent imaging showed that the crystalline coronene nano/microwires can be used as waveguide material. Both the conductivity and fluorescence of the crystalline nano/microwires change selectively in the presence of nitrobenzene vapor, a representative nitroaromatic. This suggests that the coronene nano/ microwires may be used for selective detection of explosives since many explosives belong to a group of electron-deficient nitroaromatics.
\end{abstract}

\section{Introduction}

Modern security concerns have led to a high demand for stand-off detection of trace explosive vapors in preventing possible terrorist attacks. ${ }^{1}$ Methods available for the analysis of air samples of explosives, such as ion mobility spectrometry and mass spectrometry, ${ }^{2}$ are expensive, not easily portable, time-consuming, and often require laborious sample preparation. Methods to detect explosives that rely on the interaction of sensing materials with explosives hold promise for detecting explosives with real-time response, high sensitivity and selectivity, compact size, low power requirements, and low cost. ${ }^{3}$ Recent studies have resulted in a variety of materials that change color or fluorescence characters on their interaction with explosives. ${ }^{4,5}$ However, there have been few studies on the use of conductivity changes to detect explosives, mainly due to a lack of selectivity. ${ }^{6,7}$

It has been recognized that sensors based on conductivity changes are potentially advantageous in both cost and convenience because they could eliminate the complexity and power consumption inherent to optical instruments. One strategy to enhance selectivity in detecting explosives is to develop appropriate electron-rich materials that can form a strong complex with the explosive material, due to the fact that many explosives belong to a group of electron-deficient nitroaromatics. Examples of electron-rich materials include most polycyclic aromatic hydrocarbons (PAH). ${ }^{8-10}$

To develop conductivity-change based sensors, one-dimensional (1D) nano/microstructures of electron-rich PAHs are expected to achieve higher sensitivity ${ }^{11}$ than bulky structures because of their greater surface area. Among the large family of PAHs, coronene (Figure 1) is expected to be a good candidate in the development of a conductivity-based sensor for explosives since coronene is a wellknown semiconductive material that may be used for light emitting diodes, photoconductive devices, and solar cells. ${ }^{12-14}$ Furthermore, the $\pi-\pi$ interaction between coronene molecules is stronger than that of smaller PAHs and the symmetrical structure of coronene can assist the formation and the stability of 1D nano/microstructures.

In this study, we report the synthesis and characterization of 1D coronene nano/ microwires and demonstrate our preliminary study on their potential application in detecting electron deficient chemicals, with nitrobenzene as a representative compound. We show that both the fluorescence and conductivity of the coronene nano/microwires can be used for selective detection.

\section{Materials and Methods}

Coronene was purchased from Alfa Aesar and used as received. The coronene nano/microwires were fabricated in a furnace according to a vapor deposition method reported previously. ${ }^{15}$ An amount of coronene powder was placed in a 50 -mL quartz tube. The tube was heated in a vacuum and then left to cool at room temperature. Coronene nano/microwires that had self-assembled on a glass substrate in the tube were collected for analysis.

Fluorescence spectra were obtained with a Hitachi F-7000 fluorescence spectrometer. The excitation wavelength was $325 \mathrm{~nm}$. Attenuated total reflection infrared (ATR-IR) spectra were recorded on a Smith IR microscope. The thermal analysis was obtained with a thermogravimeter/differential thermal analyzer (TG/DTA 6300, SII Nanotechnology Inc). The heating rate was $5^{\circ} \bullet \mathrm{min}^{-1}$ with a nitro-
Correspondence: Hai-Feng Ji, Department of Chemistry, Drexel University, Philadelphia, PA 19104, USA.

Tel. +1.215.895.2562 - Fax: +1.215.895.1265.

E-mail: hj56@drexel.edu

Key words: nano/microwires, coronene, sensing electron-deficient aromatics.

Received for publication: 2 September 2011. Revision received: 28 November 2011. Accepted for publication: 6 December 2011.

This work is licensed under a Creative Commons Attribution NonCommercial 3.0 License (CC BYNC 3.0).

(C) Copyright H. Wang et al., 2012

Licensee PAGEPress, Italy

Nanotechnology Development 2012; 2:e1

doi:10.4081/nd.2012.e1

gen flow $\left(50 \mathrm{~mL} \bullet \mathrm{min}^{-1}\right)$. Cross-polarized optical microscopy (CPOM) was performed on a Smith polarized microscope. A Zeiss Supra 50 VP was used to obtain scanning electron microscopy (SEM) images. A JOEL JEM2100 operated at $120 \mathrm{keV}$ was used to obtain transmission electron microscopy (TEM) images. Gas sensing experiments were performed with a microwire resistor device placed in a glass tube $20 \mathrm{~cm}$ long and $1.5 \mathrm{~cm}$ in diameter. A targeted vapor was transferred to the tube to reach a concentration of $200 \mathrm{ppm}$. Since several seconds are needed to equilibrate I-V curves and fluorescence spectra upon exposure to the chemical vapor, which may due to the small size of the nano/microwires, the curves and spectra were first collected in $\mathrm{N}_{2}$ and then collected after the device had been exposed to the chemical vapors for $30 \mathrm{~s}$.

\section{Results and Discussion}

Methods to synthesize 1D organic nanomaterials include precipitation from solutions, ${ }^{16,17}$ template synthesis, ${ }^{18}$ solid phase reaction, ${ }^{19}$ and vapor deposition..$^{20,21}$ Among these methods, vapor deposition represents an easy approach to develop 1D nanostructures. In our experiments, we developed coronene nano/microwires using a vapor deposition method reported previously ${ }^{15}$. Figure 2 shows SEM images of coronene nano/microwires when the experiment is carried out at $350^{\circ} \mathrm{C}$ for $30 \mathrm{~min}$. These nanowires have a diameter of approximately $300 \mathrm{~nm}$ and are 3-5 $\mu \mathrm{m}$ long. When the heating time increases to $5 \mathrm{~h}$, the diameter increases to approximately 1-2 $\mu \mathrm{m}$ and the length increases to approximately $5-10 \mu \mathrm{m}$. When the reac- 
tion time is longer than $5 \mathrm{~h}$, no obvious changes in size were observed.

ATR-IR spectroscopy and TG/DTA were performed to compare the molecular structure of the nanowires and the coronene powder. Figures 3 and 4 show no distinguishable difference between ATR-IR or TG/DTA of coronene nano/microwires and powder. This is expected since the $\pi-\pi$ interaction is widely presented in $\mathrm{PAH}$ molecules, even in the form of powders. However, one useful conclusion to be drawn from these results is that after the thermal heating process in our experiments, the coronene remains intact and does not undergo polymerization or decomposition.

Figure 5 shows the TEM image of a coronene nanowire. No electron diffraction pattern was observed for the nanowire structure. This may be due to that the self-assembly of coronene molecules can be readily disintegrated under high electron beam voltage, and does not confirm that the nanowire is crystalline or amorphous. The crystallinity of the 1D structures of coronene, however, can be determined from other techniques, such as polarized optical microscopy (CPOM). Figure
6A shows the brightness of CPOM images of a coronene microwire on varying the angle between the long axis of the microwire and the polarization light. Minimum brightness is seen when the long axis is parallel to the polarization light $\left(0^{\circ}\right)$. This position is called the position of extinction for the crystal. The brightness increases when the angle increases from $0^{\circ}$ to $45^{\circ}$. The brightness versus the angle (Figure $6 \mathrm{~B}$ ) shows four repeat profiles in a complete circle. Minimum brightness occurs at $0^{\circ}, 90^{\circ}, 180^{\circ}$ and $270^{\circ}$, and maximum brightness occurs at $45^{\circ}, 135^{\circ}, 225^{\circ}$, and $315^{\circ}$. Similar results were observed for coronene nanowires. The periodical change in brightness versus the angle shows the coronene nano/microwire is a birefringent crystal, ${ }^{17,22}$ i.e. the crystal has two distinct indices of refraction and it splits one ray of light into two rays.

Figure 7 shows fluorescence spectra $\left(\lambda_{\mathrm{ex}}=325 \mathrm{~nm}\right)$ of coronene nano/microwires and coronene powder. No obvious difference was observed between the fluorescence spectrum of coronene powder and microwires, suggesting they have the same molecular packing. The result is consistent with that obtained from the FTIR spectra. The fluorescence of the coronene nano/microwires is significantly brighter at the end of the wires. This is due to the light propagation along the length of the nano/microwires, the so-called outcoupling light, ${ }^{23-25}$ i.e. the light propagation along the nano/microwires ${ }^{23}$ as the coronene molecules re-absorb the emitted light. The result shows that the coronene nano/microwires may be used as waveguide material for optoelectronic applications. This optical waveguiding property indicates the high degree of molecular orientation in the nanowire, ${ }^{26}$ which is consistent with the results of cross-polarized light experiments.

Figure 8 shows the conductivity and fluorescence changes in a coronene microwire on exposure to a variety of chemicals at the same concentration. The conductivity of the microwire decreases on exposure to any of these chemical vapors, and the most dramatic decrease was when the microwire was exposed to nitrobenzene, a representative nitroaromatic. Since toluene does not have an electron-withdrawing group and dimethylformamide does not have an aromatic ring, these preliminary studies indicated that both an

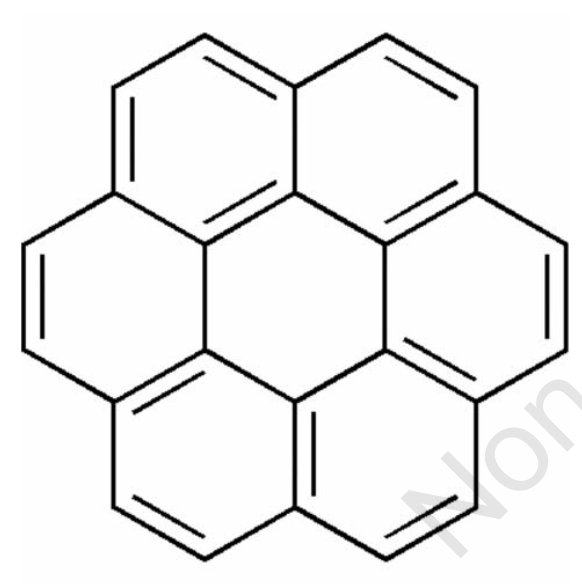

Figure 1. Structure of coronene.

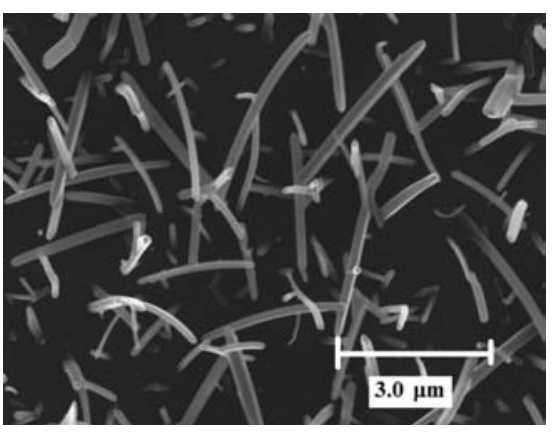

Figure 2. SEM images of coronene nanostructures formed at $350^{\circ} \mathrm{C}$ for $30 \mathrm{~min}$.

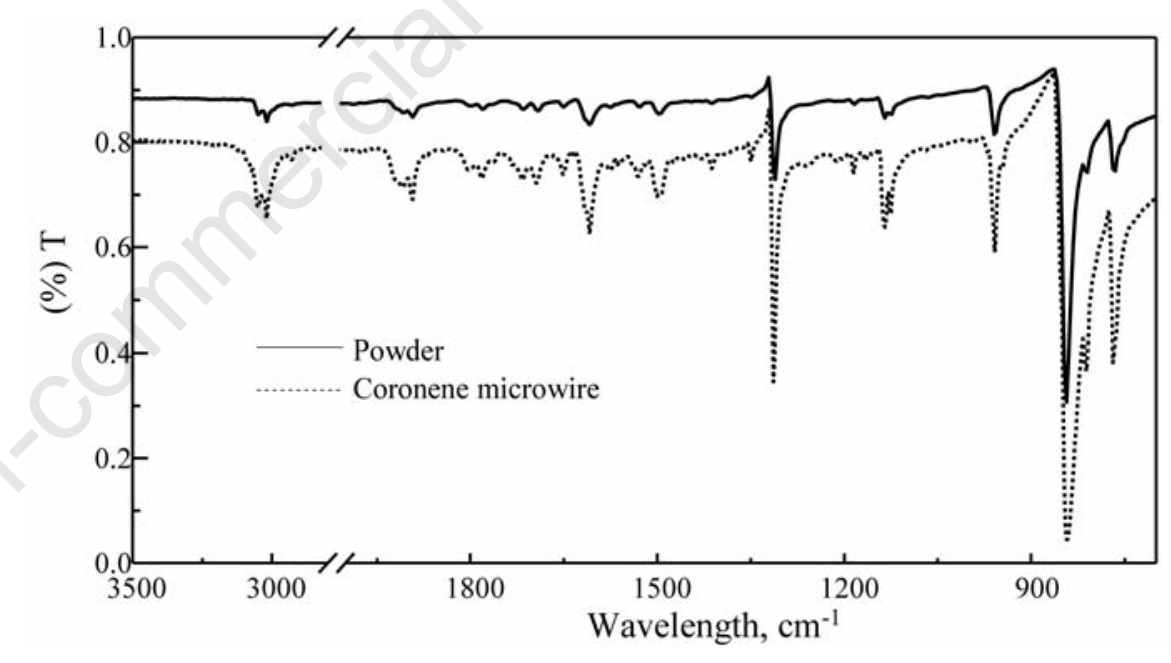

Figure 3. ATR-IR spectra of coronene powder (solid line) and nanowires (dashed line).
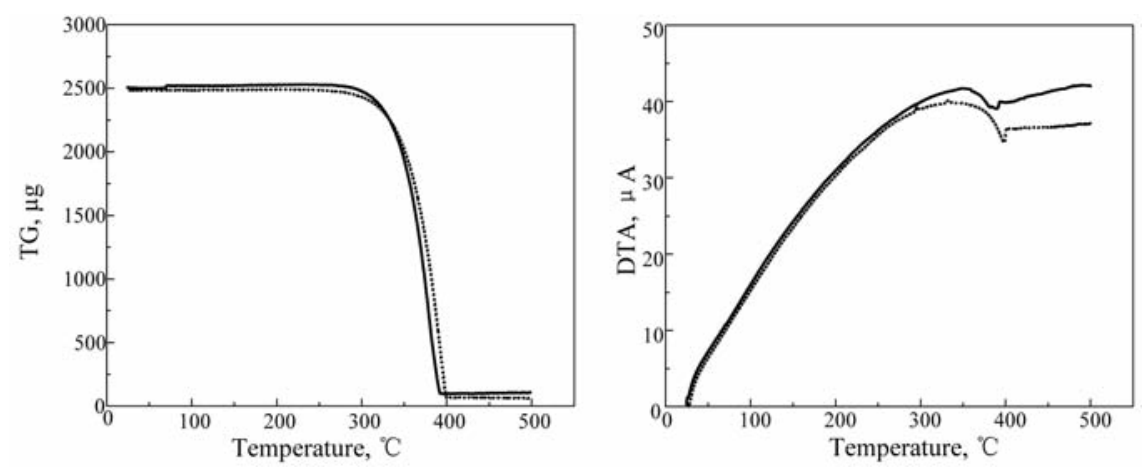

Figure 4. TG/DTA of coronene powder (soid line) and microwires (dashed line). 
aromatic ring and electron-withdrawing groups are required in order to significantly decrease the conductivity of the coronene microwire. The results suggested that nano/microwires of coronene may be used for selective detection of explosives since many explosives belong to a group of electron-deficient nitro-aromatics. In addition to the conductivity change, the fluorescence of the nanowires also showed selective change to nitrobenzene (Figure 8B). The sensing behavior of the coronene nano/microwires will be further studied, and the sensing behavior and sensing mechanism will be reported as soon as these are available. However, it is most likely that the changes in current and fluorescence can be attributed to both surface binding on and diffusion of nitrobenzene in the nanowires. ${ }^{27-30}$ Coronene is an electron-rich ptype material, which can form a strong com-

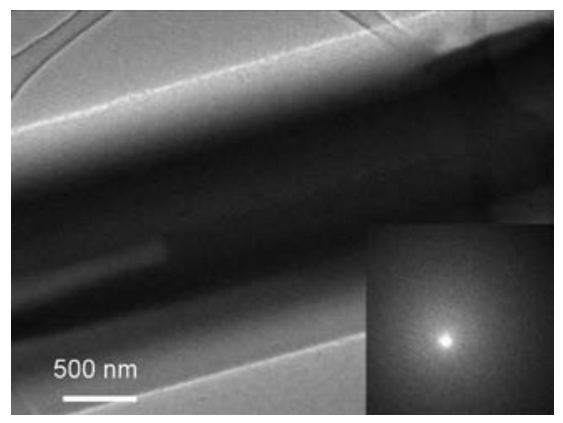

Figure 5. TEM images of a coronene nanowire. The corresponding electron diffraction pattern of a solid nanowire is shown in the insert.
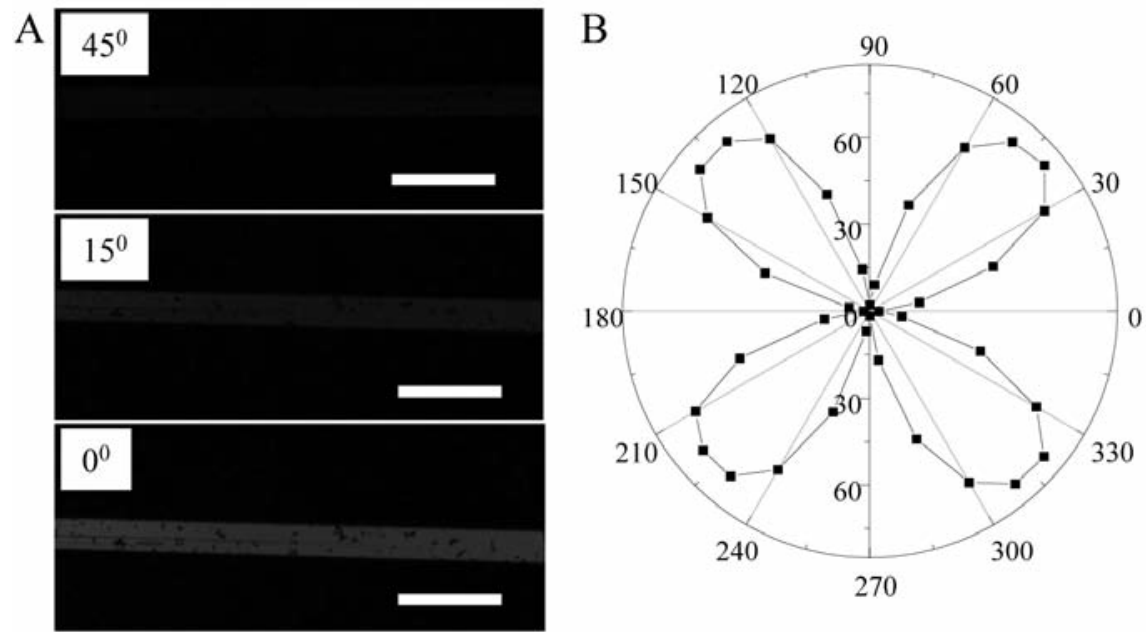

Figure 6. (A) Cross-polarized optical microscopy (CPOM) images of a coronene microwire. Scale bar is $3 \mu \mathrm{m}$. (B) Brightness versus the angle between the polarization direction of the light and the long (optical) axis of the coronene microwire.
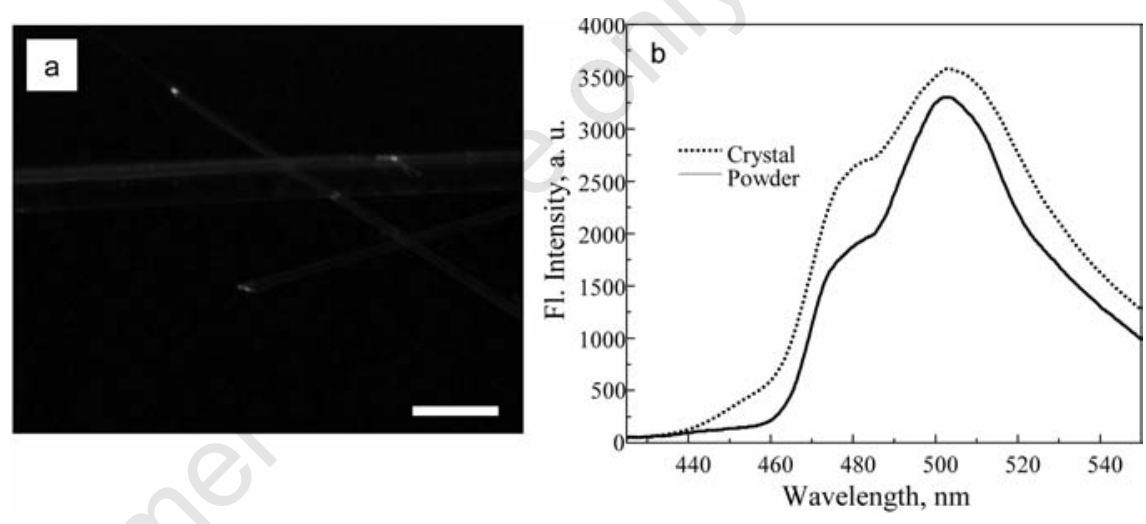

Figure 7. (A) Flourescence microscopy images of coronene microwires, exited with 510$550 \mathrm{~nm}$ UV. Scale bar is $5 \mu \mathrm{m}$. (B) Emission spectra of coronene powder (dashed line) and microwires (solid line).
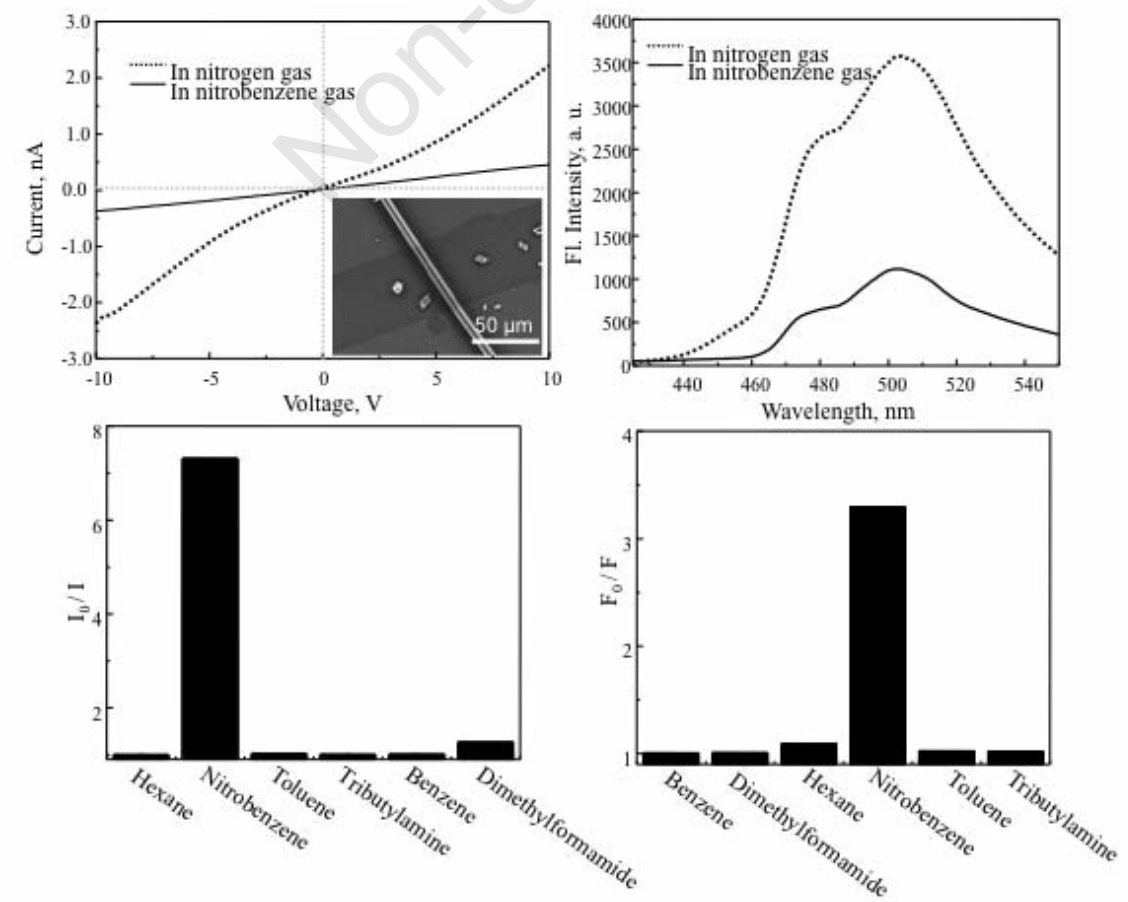

Figure 8. Effect of chemical vapors. (A) Effect on conductivity: a single coronene microwire device with sputtered Al on both ends. (B) Effect on fluorescence of a single coronene microwire $(10 \sim 20 \mu \mathrm{m}$ in diameter). $I_{0} / I$ and $F_{0} / F$ are the ratios of current and fluorescence, respectively, in the vacuum to those in the presence of chemical vapors at the same concentration $(200 \mathrm{ppm})$. 
plex with nitrobenzene due to the fact that nitrobenzene moiety is electron-deficient. This feature means coronene can participate in $\pi-\pi$ interactions with nitrobenzene, which decrease the hole transportation and quench the fluorescence of the excitons of the nanowires.

\section{Conclusions}

In this study, 1D crystalline coronene nano/microwires were synthesized and characterized. Cross-polarized and fluorescence images show coronene nano/microwires have a crystalline structure and a high degree of molecular orientation. Conductivity and fluorescence of the coronene nano/microwires change selectively in the presence of nitrobenzene, indicating its potential to detect explosive chemicals. The easy synthesis method and its high sensitivity make it competitive in commercial applications.

Since ordered nanostructures can be prepared from a large pool of PAH molecules, ${ }^{31,32}$ and heterocyclic PAHs with $\mathrm{N}, \mathrm{S}$, or $\mathrm{O}$ in the rings, or PAHs with $\mathrm{OH}, \mathrm{NH}_{2}$, or $\mathrm{C}=0$ groups, we expect that the semiconductive and optical properties of these nanostructures can be tuned by the selection of different PAH molecules, i.e. nanostructures from various $\mathrm{PAH}$ materials have different bandgaps or belong to different types, such as n or $\mathrm{p}$ type materials. We focused on the characterization of the nano/microwires in this study and the sensing performance of these nano/microwires and other nanostructures to explosives will be investigated and reported in the future. Other potential applications of these nanostructures include nanoelectronics and photoelectronics devices, and solar cells.

\section{References}

1. McQuade DT, Pullen AE, Swager TM. Conjugated polymer-based chemical sensors. Chem Rev 2000;100:2537-74.

2. Popov IA, Chen H, Kharybin ON, et al. Detection of explosives on solid surfaces by thermal desorption and ambient ion/molecule reactions. Chem Commun 2005;15:1953-5.

3. Miller JN. Some recent advances in fluorescence spectroscopy. Anal Lett 2006;39: 851-62.

4. Sanchez JC, Urbas SA, Toal SJ, et al. Catalytic hydrosilylation routes to divinylbenzene bridged silole and silafluorene polymers. Applications to surface imaging of explosive particulates. Macromolecules 2008;41:1237-45.

5. Yang JS, Swager TM. Fluorescent porous polymer films as TNT chemosensors: electronic and structural effects. J Am Chem Soc 1998;120:11864-73.

6. Yinon, J. Detection of explosives by electronic noses Anal Chem 2003;75:99A-105A.

7. Matzger AJ, Vaid TP, Lewis NS. Vapor sensing with arrays of carbon black-polymer composites. Proc SPIE Int Soc Opt Eng 1999;3710:315-20.

8. Lee HM, Moon H, Kim HS, et al. Abrupt heating-induced high-quality crystalline rubrene thin films for organic thin-film transistors. Organ Electron 2011;12:144653.

9. Spalenka JW, Paoprasert P, Franking R, et al. Molecular control of pentacene/ZnO photoinduced charge transfer. Appl Phys Lett 2011;98:103303.

10. Khan HU, Roberts ME, Knoll W, et al. Pentacene based organic thin film transistors as the transducer for biochemical sensing in aqueous media. Chem Mater 2011;23:1946-53.

11. Engel Y, Elnathan R, Pevzner A, et al. Supersensitive detection of explosives by silicon nanowire arrays. Angew Chem Int Ed 2010;49:6830-5.

12. Xiao J, Yang H, Yin Z, et al. Preparation, characterization, and photoswitching/ light-emitting behaviors of coronene nanowires. J Mater Chem 2011;21:1423-7.

13. Li J, Kastler M, Pisula W, et al. Organic bulk-heterojunction photovoltaics based on alkyl substituted discotics. Adv Mater 2007;17:2528-33.

14. Gorodetsky AA, Chiu CY, Schiros T, et al. Reticulated heterojunctions for photovoltaic devices. Angew Chem Int Ed 2010; 49:7909.

15. Ji HF, Majithia R, Yang X, et al. Self-assem- bly of perylenediimide and naphthalenediimide nanostructures on glass substrates through deposition from the gas phase. J Am Chem Soc 2008;130:10056-7.

16. Zhao M, Wang S, Bao Q, et al. A simple, high yield method for the synthesis of organic wires from aromatic molecules using nitric acid as the solvent. Chem Commun 2011;47:4153-5.

17. Niu Q, Zhou Y, Wang L, et al. A solution process for size-controlled growth and transfer of organic nanostructures with manufacture scalability. Langmuir 2010; 26:5213-6.

18. Lee JK, Koh WK, Chae, WS, et al. Novel synthesis of organic nanowires and their optical properties. Chem Commun 2002; 38:138-9.

19. Liu H, Li Y, Xiao S, et al. Synthesis of organic one-dimensional nanomaterials by solid-phase reaction. J Am Chem Soc 2003;125:10794-5.

20. Borras A, Aguirre M, Groening 0, et al. Synthesis of supported single-crystalline organic nanowires by physical vapor ceposition Chem Mater 2008;20:7371-3.

21. Xiao K, Rondinone AJ, Puretzky AA, et al. Growth, patterning, and one-dimensional electron -transport properties of selfassembled Ag-TCNQF4 organic nanowires. Chem Mater 2009;21:4275-81.

22. Lu GH, Li LG, Li SJ, et al. Constructing thin polythiophene film composed of aligned lamellae via controlled solvent vapor treatment. Langmuir 2009;25:3763-8.

23. Zhao YS, Fu HB, Hu FQ, et al. Tunable emission from binary organic one-dimensional nanomaterials: an alternative approach to white-light emission. Adv Mater 2008;20:79-83.

24. Balzer F, Bordo VG, Simonsen AC, et al. Isolated hexaphenyl nanofibers as optical waveguides. Appl Phys Lett 2003;82:10-2.

25. Takazawa K, Kitahama Y, KimuraY, et al. Optical waveguide self-assembled from organic dye molecules in solution. Nano Lett 2005;5:1293-6.

26. Zhao YS, Zhan P, Kim J, et al. Patterned growth of vertically aligned organic nanowire waveguide arrays. ACS Nano 2010;4:1630. 